\title{
Utilización de Servicios Médicos por una Población Desplazada en Bucaramanga, Colombia
}

Myriam Ruiz-Rodriguez ${ }^{1}$, Sergio López-Moreno ${ }^{2}$, Leticia Ávila-Burgos ${ }^{3}$ y Naydú Acosta-Ramirez ${ }^{4}$

${ }^{1}$ Enfermera. M. Sc. en Sistemas de Salud. Departamento de Salud Pública, Escuela de Medicina, Universidad Industrial de Santander (UIS), Bucaramanga, Colombia. E-mail: myriam@uis.edu.co ${ }^{2}$ Médico Ph.D. en Epidemiología. División de Ciencias Biológicas y de la Salud. Universidad Autónoma Metropolitana, Unidad Xochimilco.Ciudad México, México.E-mail: slopez@correo.xoc.uam.mx ${ }^{3} \mathrm{Ph}$.D. en Economía. Centro de investigación en Sistemas de Salud (CISS). Instituto Nacional de Salud Pública. Cuernavaca, México. E-mail: lavila@insp.mx

${ }^{4} \mathrm{Ph}$.D en Salud Pública. Centro de Proyectos para el Desarrollo (CENDEX). Pontificia Universidad Javeriana, Bogotá, Colombia. E-mail: nacosta@javeriana.edu.co

Recibido 13 Abril 2006/Enviado para Modificación 6 Agosto 2006/Aceptado 21 Septiembre 2006

RESUMEN

Objetivo Analizar los factores y barreras asociadas a la utilización de servicios médicos por la población desplazada, como consecuencia del conflicto armado colombiano, residente en Bucaramanga, Colombia.

Material y Métodos A partir de datos procedentes del estudio "Diagnóstico de Salud de las Poblaciones Desplazadas y no Desplazadas en Bucaramanga y su área Metropolitana" diseñado y ejecutado por la Universidad Industrial de Santander y financiado por la Organización Panamericana de la Salud, se analizó la utilización de los servicios médicos en individuos mayores de 15 años de edad de acuerdo con el modelo de Andersen y Newman y se evaluó el efecto de los factores asociados mediante regresión logística múltiple.

Resultados Una de cada cinco personas que enfermó en los quince días previos a la encuesta usó los servicios médicos. Este porcentaje es menor a los encontrados en la población general de Colombia. Pertenecer al régimen contributivo de seguridad social, el antecedente de visita a los servicios de salud y ser originario de la misma región se encontraron asociados a la mayor utilización de los servicios. Los desplazados que tienen derecho al seguro de salud subsidiado no presentaron diferencias en el uso de los servicios con respecto a los que no cuentan con seguro. La distancia a la institución de salud fue otra barrera para la utilización de servicios ( $R M=0,64$; IC 0,42-0,97). Conclusión Existe un uso de servicios médicos diferencial entre los desplazados, determinado por sus condiciones de aseguramiento y su capacidad económica.

Palabras Clave: Atención médica, enfermedad, conflictos armados (fuente: DeCS, BIREME). 


\section{ABSTRACT Displaced people's healthcare use in Bucaramanga, Colombia}

Objective Analysing the factors and barriers associated with the population displaced by armed conflict using medical services in Bucaramanga, Colombia. Material and Methods Data from the "Diagnosing the health of both displaced and non-displaced populations in Bucaramanga and its metropolitan area" study, designed and executed by the Industrial University of Santander and financed by the Pan-American Health Organisation, analysed medical service use in individuals aged over 15 years. This agreed with Andersen and Newman's model and evaluated the effect of associated factors by means of multiple logistical regression

Results One out of each five people who became ill during the fifteen days prior to the survey had used the medical services. This percentage was smaller than that found in the Colombian population as a whole. The type of social security regime (contributory health insurance), previous service use and being from the region were associated with greater service use. Displaced people having subsidised health insurance did not present differences in service use compared to those who had no access to such insurance. The distance from dwelling to institution was another barrier against using health services (RM=0.64; IC 0.42-0.97).

Conclusions There was differential medical service use amongst the displaced population, determined by their health insurance coverage and economic position.

Key Words: Mdical care, disease, armed conflict (source:MeSH, NLM).

$\mathbf{E}$ I desplazamiento poblacional forzado se ha convertido en los años recientes en una importante preocupación en el campo de la salud pública, debido a sus repercusiones en la salud física y mental de las poblaciones afectadas por el desplazamiento. En el caso de Colombia, el éxodo interno de colombianos por el conflicto armado ha ido aumentando desde 1985. A partir de esta fecha, las autoridades calculan en millón y medio las personas desplazadas (1). Otras fuentes calculan que en el año 2003 este número alcanzó los tres millones. Esta cifra representa aproximadamente el $12 \%$ de los 20 a 25 millones de desplazados a nivel mundial estimados por la Organización de las Naciones Unidas-ONU (2). Para el año 2001 se estimó que 50 \% de los desplazados colombianos se concentraron en 16 ciudades, entre las que figuraba Bucaramanga, capital del Departamento de Santander (3).

La magnitud del impacto de la violencia y el desplazamiento obligó al gobierno a formular desde 1997 una legislación y un plan nacional para 
promover, proteger y defender los derechos humanos de ese grupo poblacional (4). A pesar de la existencia de normatividad orientada a garantizar el acceso a los servicios de salud por la población desplazada, las tasas de utilización de servicios de salud son bajas y se han reportado barreras para el acceso a los servicios (5-7).

El presente estudio tiene como propósito identificar y analizar los factores asociados al proceso de uso de servicios médicos por la población desplazada ubicada en asentamientos en el área metropolitana de Bucaramanga (AMB).

\section{MATERIAL Y MÉTODOS}

Los datos provienen del estudio transversal denominado "Diagnóstico de Salud de las Poblaciones Desplazadas y no Desplazadas en Bucaramanga y su área Metropolitana", diseñado y ejecutado por la Universidad Industrial de Santander de Bucaramanga-UIS, financiado por la Organización Panamericana de la Salud -OPS.

El muestreo del estudio mencionado fue aleatorio estratificado, donde cada asentamiento se consideró como un estrato, y representaba a cada uno de los municipios que conforman el AMB: estrato 1: Bucaramanga; estrato 2: Floridablanca; estrato 3: Girón, y estrato 4: Piedecuesta. El marco muestral fue el total de familias en situación de desplazamiento ubicadas en el AMB, identificadas por la Red de Solidaridad Social (RSS).

Para el cálculo de la muestra se consideró $50 \%$ de prevalencia de enfermedad, una precisión de $6 \%$ y un nivel de confianza de $95 \%$. Siguiendo el método de afijación proporcional se determinaron tamaños muestrales en cada estrato, de manera que se mantuviera el mismo peso relativo dado por el número de familias en cada estrato frente al total de ellas en la población. Así el total de la muestra fue de 257 familias en situación de desplazamiento, con un promedio de 4,4 personas por familia, lo cual representa un total de 1131 personas en situación de desplazamiento.

La población estudiada en la presente investigación corresponde a 181 personas de 15 años y más de edad ubicadas en asentamientos del AMB, que fueron desplazadas por conflicto armado y que reportaron haber tenido algún problema de salud 15 días previos a la encuesta. Esta población representa 30,3 \% del total de la muestra del estudio principal. 
La utilización de servicios de atención médica ante la presencia de enfermedad en los 15 días previos a la aplicación de la encuesta se consideró como la variable dependiente. Como variables independientes se analizaron factores predisponentes, acorde con el marco teórico y revisiones de análisis empíricos del modelo de Andersen (8-11) estudiando los siguientes indicadores: edad, sexo, tamaño de la familia, escolaridad, ocupación, estado civil, procedencia, antecedentes de enfermedad (morbilidad no reciente) y utilización de servicios de atención médica en el último año; además se adicionó una variable especifica que describe el grupo poblacional en estudio, como es el motivo de desplazamiento.

En el grupo de las variables habilitadoras se indagaron variables especificas del sistema colombiano de salud, como la cobertura del SGSSS; el tipo de régimen de afiliación al SGSSS; la tenencia de documentos que facilitan el acceso a servicios de salud (tales como el carnet del SISBEN y la carta de la Red de Solidaridad, la cual los identifica como población desplazada); además se incluyeron variables como el tiempo de permanencia en el asentamiento; la residencia en otros asentamientos; el municipio de ubicación del asentamiento y la distancia en kilómetros de la institución pública de salud de primer nivel de atención más cercana al asentamiento. El tipo de enfermedad (crónica/no crónica) sufrido durante los últimos 15 días previos al momento de la encuesta se utilizó como indicador de necesidad.

Mediante análisis univariado se estimaron las frecuencias simples y los porcentajes. En el bivariado se estimaron las diferencias de proporciones entre la población de utilizadores y no utilizadores de servicios médicos considerando un valor de $\mathrm{p} £ 0.10$ como estadísticamente relevante para seleccionar las variables independientes relacionadas con el uso de servicios médicos, y calculando la razón de momios cruda (RMc) como medida de asociación. Por último, se realizó un modelo de regresión logística, donde á $<0.05$ y un intervalo de confianza (IC) de $95 \%$ fueron definidos a priori como criterio de significancia. Al modelo final ingresaron las variables que presentaron un nivel de p 0.25 , y aquellas de relevancia teórica como edad y tiempo de permanencia en el asentamiento.

La muestra ( $\mathrm{n}=1903$ ) fue ponderada de acuerdo a la probabilidad de selección de las familias por municipio. Se consideró a cada municipio como un estrato y a la familia como la unidad primaria de muestreo, de tal manera que se corrigiera el efecto de la posible correlación intraclase entre los individuos que pertenecen a una misma familia. Debido a que actualmente no existen paquetes de software para evaluar el ajuste de modelos logísticos en muestras complejas, no se realizó 
la evaluación del modelo final (12). Todos los cálculos se realizaron con la muestra estratificada, ponderada y con la familia como conglomerado, utilizando el programa STATA versión 8.0, que toma en cuenta el diseño del muestreo y la ponderación.

\section{RESULTADOS}

En la Tabla 1 se observan las principales variables sociodemográficas de la población desplazada. Esta población se caracteriza porque se ubica predominantemente en el municipio de Bucaramanga (52,5\%), presenta un alto porcentaje de mujeres (62.4\%), y bajos niveles de escolaridad y aseguramiento en salud.

Los entrevistados pertenecían a familias cuyo promedio de integrantes era de $4,9 \pm 1,8$. El tiempo de permanencia en el asentamiento, en promedio, fue de 27,8 meses. La distancia más corta en kilómetros desde el asentamiento hasta la institución pública de salud de primer nivel de atención fue la de Bucaramanga (1,2 km.), y la más larga, la de Piedecuesta (5 km.). Al interrogar sobre historia de contacto con los servicios de atención médica, 64,6\% de los entrevistados refirieron que utilizaron servicios médicos durante el último año.

De todos los síntomas manifestados por los interrogados según el sistema del cuerpo involucrado, 54,5\% se concentran en cuatro sistemas: respiratorio, 22,9\%; osteomuscular y del tejido conectivo, 13,3\%; digestivo y abdomen, 9,9 $\%$; y del conocimiento, percepción, el estado emocional y de la conducta, 8,5 $\%$. Al clasificar la sintomatología reportada en tipo de morbilidad-crónica y no crónica- se encontró que 11,2 \% eran crónicas. Del total de éstas, 60 \% se presentó en hombres.

El porcentaje de utilización de servicio médico fue de 19,9\%. Entre las variables que presentaron mayor uso y que estuvieron asociadas estadísticamente en el análisis bivariado, destacan: tipo de enfermedad (crónica) con 35 \%; zona de procedencia (ser del área urbana) con 33 \% y escolaridad (tener siete años o más de escolaridad) con 30,4\%. Al analizar la asociación entre atención médica y tipo de régimen se encontró que 42,8 \% de los desplazados enfermos afiliados al régimen contributivo recibieron atención médica, mientras sólo lo hizo 15,4 \% de los del régimen subsidiado y $16,3 \%$ de los vinculados. 
202 REVISTA DE SALUD PÚBLICA · Volumen 8 (3), Noviembre 2006

Tabla 1. Características sociodemográficas de la población desplazada de 15 y más años de edad. Área metropolitana de Bucaramanga, 2003

\begin{tabular}{|c|c|c|c|c|}
\hline Variables & $\begin{array}{c}\text { Femenino } \\
\mathrm{n}=1187^{*} \\
\%\end{array}$ & $\begin{array}{c}\text { Masculino } \\
n=716^{\star} \\
\%\end{array}$ & $\begin{array}{c}\text { Total } \\
\mathrm{n}=1 \text { 903* } \\
\%\end{array}$ & $\begin{array}{c}\mathrm{X}^{2} \dagger \\
\text { Valor } p\end{array}$ \\
\hline Edad & & & & 0.13 \\
\hline $15-24$ & 16,7 & 8,7 & 25,4 & \\
\hline $25-34$ & 12,2 & 11,2 & 23,4 & \\
\hline $35-44$ & 14,9 & 3,8 & 18,7 & \\
\hline $45-54$ & 8,2 & 6,1 & 14,3 & \\
\hline 55 y más & 10,4 & 7,8 & 18,2 & \\
\hline Años de Escolaridad & & & & 0.10 \\
\hline Ninguno & 14,8 & 8,8 & 23,6 & \\
\hline $1-3$ & 15,8 & 15,1 & 30,9 & \\
\hline $4-6$ & 22,0 & 10,9 & 32,9 & \\
\hline 7 y más & 9,9 & 2,7 & 12,6 & \\
\hline Estado civil & & & & 0.16 \\
\hline Con pareja & 41,8 & 28,9 & 70,7 & \\
\hline Sin pareja & 20,6 & 8,7 & 29,3 & \\
\hline Ocupación & & & & 0.00 \\
\hline Trabajadores calificados & 4,2 & 10,5 & 14,7 & \\
\hline $\begin{array}{l}\text { Trabajadores no } \\
\text { calificados }\end{array}$ & 12,9 & 13,3 & 26,2 & \\
\hline Amas de casa/estudiante & 37,6 & 3,2 & 40,8 & \\
\hline Desempleados & 7,8 & 10,5 & 18,3 & \\
\hline $\begin{array}{l}\text { Tipo de régimen de afiliación } \\
\text { a la seguridad social }\end{array}$ & & & & 0.93 \\
\hline Contributivo & 2,2 & 1,7 & 3,9 & \\
\hline Subsidiado & 8,8 & 5,5 & 14,3 & \\
\hline Vinculado & 51,4 & 30,4 & 81,8 & \\
\hline Identificación como desplazado & & & & 0.22 \\
\hline $\mathrm{Si}$ & 50,6 & 27,5 & 78,1 & \\
\hline No & 11,8 & 10,1 & 21,9 & \\
\hline Motivo del desplazamiento & & & & 0.29 \\
\hline Miedo y amenaza verbal & 47,7 & 30,0 & 77,7 & \\
\hline Muerte de un familiar & 12,5 & 4,9 & 17,4 & \\
\hline Agresión física & 2,2 & 2,7 & 4,9 & \\
\hline Zona de procedencia & & & & 0.80 \\
\hline Urbana & 14,9 & 8,3 & 23,2 & \\
\hline Rural & 47,5 & 29,3 & 76,8 & \\
\hline Región del país de donde procede & & & & 0.48 \\
\hline Santander & 37,5 & 20,6 & 58,1 & \\
\hline Otra & 24,9 & 17,0 & 41,9 & \\
\hline Permanencia en el asentamiento & & & & 0.99 \\
\hline 0-12 meses & 13,0 & 7,8 & 20,8 & \\
\hline 13 meses y más & 49,4 & 29,8 & 79,2 & \\
\hline
\end{tabular}

*Muestra ponderada; $† X^{2}$ de Pearson

En la Tabla 2 aparece el modelo de regresión logística múltiple de utilización de servicios médicos. Los factores explicativos más importantes asociados con la utilización de los servicios de atención médica fueron los siguientes: i) estar afiliado al Sistema General de Seguridad Social en Salud a través del régimen 
contributivo; ii) haber asistido a los servicios de atención médica durante el último año; iii) contar con un mayor tiempo de permanencia en el asentamiento; iv) provenir de la misma región del país y v) estar a menor distancia de la institución de salud. Todos estos factores estuvieron asociados de manera estadísticamente significativa a la mayor posibilidad de utilizar servicios de salud $(\mathrm{p}<0.05)$.

Tabla 2. Factores asociados a la utilización de servicios de atención médica por población desplazada. Área metropolitana de Bucaramanga, 2003

\begin{tabular}{|c|c|c|c|}
\hline Factores & $\begin{array}{c}\text { RM } \\
\text { Ajustada* }\end{array}$ & IC $95 \%$ & Valor $p$ \\
\hline \multicolumn{4}{|l|}{ Predisponentes } \\
\hline \multicolumn{4}{|l|}{ Sexo } \\
\hline Hombre & 0,409 & $0,16-1,01$ & 0.053 \\
\hline Mujer & 1 & & \\
\hline Edad & 0,995 & $0,97-1,02$ & 0.763 \\
\hline \multicolumn{4}{|l|}{ Años de escolaridad } \\
\hline 4 y más & 1,425 & $0,61-3,32$ & 0.410 \\
\hline $0-3$ & 1 & & \\
\hline \multicolumn{4}{|l|}{ Zona de procedencia } \\
\hline Urbana & 2,538 & $0,99-6,49$ & 0.052 \\
\hline Rural & 1 & & \\
\hline \multicolumn{4}{|l|}{ Región del país de donde proceden } \\
\hline Santander & 3,159 & $1,15-8,66$ & 0.026 \\
\hline Otra & 1 & & \\
\hline \multicolumn{4}{|l|}{$\begin{array}{l}\text { Historia de utilización de servicios médicos } \\
\text { durante el ultimo año }\end{array}$} \\
\hline Sí & 3,901 & $1,15-13,18$ & 0.029 \\
\hline No & 1 & & \\
\hline \multicolumn{4}{|l|}{ Habilitadores } \\
\hline \multicolumn{4}{|l|}{$\begin{array}{l}\text { Tenencia de documento que lo identifica como } \\
\text { desplazado }\end{array}$} \\
\hline Sí & 1,893 & $0,54-6,54$ & 0.310 \\
\hline No & 1 & & \\
\hline \multicolumn{4}{|l|}{$\begin{array}{l}\text { Tipo de régimen de afiliación al sistema de } \\
\text { salud }\end{array}$} \\
\hline Subsidiado & 1,159 & $0,33-4,02$ & 0.815 \\
\hline Contributivo & 10,093 & $1,95-52,13$ & 0.006 \\
\hline Vinculado & 1 & & \\
\hline \multicolumn{4}{|l|}{ Tiempo de permanencia en el asentamiento } \\
\hline 13 meses y más & 3,285 & $1,16-9,29$ & 0.0251 \\
\hline 0-12 meses & 1 & & \\
\hline $\begin{array}{l}\text { Distancia en } \mathrm{km} \text { desde el asentamiento hasta } \\
\text { la institución de salud de primer nivel más } \\
\text { cercana }\end{array}$ & 0,644 & $0,42-0,97$ & 0.037 \\
\hline \multicolumn{4}{|l|}{ Necesidad de atención } \\
\hline \multicolumn{4}{|l|}{ Tipo de enfermedad reportada } \\
\hline Crónica & 3,522 & $0,97-12,69$ & 0.054 \\
\hline No crónica & 1 & & \\
\hline
\end{tabular}

*Razón de Momios ajustada por todas las variables incluidas en el modelo 


\section{DISCUSIÓN}

Nuestros resultados indican que una de cada cinco personas que enfermó en los quince días previos a la encuesta usó los servicios médicos. Este porcentaje de utilización es menor a los encontrados en la población general de Colombia (13) y mayor en relación con los encontrados en población desplazada; así, este es superior al reportado en el estudio de Cartagena (5).

En nuestro estudio los factores predisponentes, en especial los de tipo sociodemográfico, como la edad, el tamaño de la familia, la escolaridad, la ocupación y el estado civil no se asociaron con la utilización de servicios médicos; otro factor considerado típicamente como predisponente, como los antecedentes previos de enfermedad, tampoco estuvo asociado con esta utilización. Estos hallazgos difieren de lo informado en estudios hechos en población no desplazada, los cuales han mostrado que estos factores afectan la utilización de los servicios médicos, aunque la dirección del efecto depende mucho del país, el sistema de salud y el tipo de población estudiada (14).

Dos factores predisponentes presentaron asociación con la utilización de servicios médicos: uno de ellos es específico de la población desplazada-región del país de donde huyeron por el conflicto armado $3 / 4$ y el otro, de carácter general, es el antecedente de uso de los servicios médicos por estar enfermo durante el último año. En cuanto a la región de origen, provenir del departamento de Santander se asoció a una mayor utilización de los servicios de salud. Aparentemente, residir en el mismo departamento hace que las personas compartan elementos relacionados con la territorialidad, la cultura y, particularmente, con una misma Dirección Territorial de Salud, lo cual le da cierta familiaridad con el funcionamiento del sistema de salud.

La distancia del asentamiento a la institución de primer nivel de atención afectó la utilización de servicios médicos. En general, los estudios realizados en países con una razonable red de atención no encuentran un efecto significativo (14). La significancia de esta variable indica que aunque los desplazados estén ubicados en asentamientos urbanos del AMB, persisten problemas de accesibilidad geográfica. El hecho de tener identificación como desplazado no se asoció estadísticamente con la utilización de servicios médicos. Este resultado puede sugerir que el desplazado no percibe ventaja al identificarse como tal a la hora de solicitar servicios médicos, o bien, que existen dificultades para verificar esta identificación por parte de las instituciones de salud, tal como evidenció el estudio de Mogollón (7). 
Es importante resaltar que la variable tipo de régimen de afiliación al SGSSS fue la que presentó la mayor fuerza de asociación entre todas las variables estudiadas. Este resultado nos muestra que existe un uso de servicios médicos diferencial por tipo de aseguramiento. Las personas afiliadas al régimen contributivo tuvieron una mayor posibilidad de uso que las no afiliadas. En este aspecto hay que destacar que debido a las características socioeconómicas de la población estudiada -de extrema pobreza-hay pocos casos de afiliación a dicho régimen en el grupo estudiado, lo cual podría llevar a una sobreestimación de la RM.

Las personas afiliadas al régimen subsidiado no presentaron diferencias de uso con respecto a las no afiliadas. Este hallazgo sugiere la existencia de factores que ostensiblemente afectan la operación del régimen subsidiado, y que repercuten en la utilización de los servicios, incluidos los de atención médica. Estudios realizados en Colombia aportan evidencias de este hecho $(13,15,16)$ Por ejemplo, Plazas y colaboradores (15) clasifican en tres grupos las dificultades de operación del régimen subsidiado: a) deficiente capacidad institucional, b) deficientes canales de información, y c) inadecuado flujo de recursos.

Entre las posibles limitaciones de nuestra investigación, además de las inherentes al tipo de estudio (17), figura el número pequeño de observaciones que ingresaron al modelo multivariado, debido a la conformación de subgrupos de la muestra total-enfermos y no enfermos; enfermos utilizadores y enfermos no utilizadores-. Esta situación podría explicar la amplitud de los IC hallados e impedir tener significancia estadística en algunas de las variables, no porque no haya asociación, sino por el tamaño de la muestra.

En conclusión, este estudio aporta evidencia sobre los factores que favorecen u obstaculizan el uso de servicios médicos por población desplazada, y destaca el papel que el aseguramiento tiene a la hora de utilizar servicios. Medidas que incrementen la cobertura y garanticen un funcionamiento adecuado de los regímenes de afiliación deben ser implementadas por las autoridades locales de salud para disminuir la inequidad en el uso de servicios en este tipo de población •

\section{REFERENCIAS}

1. Consultoría para los Derechos Humanos y el Desplazamiento, CODHES. ¿Desplazados sin salida? Boletín 46. Bogotá: CODHES; 2003.

2. Global IDP project. 25m internally displaced by conflict. Geneva: Global IDP project; 2002. 
206 REVISTA DE SALUD PÚBLICA · Volumen 8 (3), Noviembre 2006

3. Consultoría para los Derechos Humanos y el Desplazamiento, CODHES. Aspectos críticos de la política de atención a la población desplazada 1998-2002. Bogotá: CODHES; 2002.

4. República de Colombia. Consejo Nacional de Seguridad Social. Acuerdos No. 59 y 85 de 1997. Bogotá: Presidencia de la República; 1997.

5. Cáceres D, Izquierdo V, Mantilla L, Jara J, Velandia M. Perfil epidemiológico de la población desplazada por el conflicto armado interno del país en un barrio de Cartagena, Colombia, 2000. Biomédica 2002;22:425-44.

6. Organización Panamericana de la Salud, OPS. Serie salud y desplazamiento en Colombia. Módulo 1-3. Bogotá: Universidad de Antioquia; 2005.

7. Mogollón A, Vásquez L, García M. Necesidades en salud de la población desplazada por conflicto armado en Bogota. Rev Esp Salud Pública 2003; 77:257-66.

8. Andersen R, Newman J. Societal and individual determinants of medical care utilization in the United States. Milbank Memorial Fund Quarterly 1973; 51:91124.

9. Aday LA, Andersen R. A framework for the study of access to medical care. Health Services research, 1974; 9: 208-20.

10. Andersen R, McCutcheon A, Aday LA, Chiu GY, Bell R. Exploring dimensions of access to medical care. Health Services Research, 1983; 18 (1): 49-74.

11. Andersen R. Revisiting the behavioral model and access to medical care: does it matter? Journal of Health and Social Behavior 1995; 36: 1-10.

12. Hosmer D, Lemeshow S. Applied logistic regression. Segunda edición, New York, John Wiley \& Sons, INC.; 2000 pp. 211-221.

13. Céspedes-Londoño J, Jaramillo-Pérez I, Castaño-Yepes R. Impacto de la reforma del sistema de seguridad social sobre la equidad en los servicios de salud en Colombia. Cad. Saude Pública 2002; 18(4):1003-24.

14. Mendoza-Sassi R, Beria J. Utilización de los servicios de salud: una revisión sistemática sobre los factores relacionados. Cad Saude Publica 2001; 17(4):819-32.

15. Plaza B, Barona B, Hearst N. Managed competition for the poor poorly managed competition? Lessons from the Colombian health reform experience. Health Policy and Planning 2001; 16(Suppl 2):44-51.

16. Céspedes J, Jaramillo I, Martinez S, Olaya S, Reynales J, Uribe C., et al. Efectos de la reforma de la Seguridad Social en Salud en Colombia sobre la equidad en el acceso y la utilización de servicios de salud. Rev. Salud Publica-Colombia 2000; 2(2):145-64.

17. Hernández B, Velasco-Mondragón HE. Encuestas transversales. Salud Pública de México 2000; 42(5):447-55. 